\title{
Exploring the Impact of Avatar Color on Game Experience in Educational Games
}

\author{
Dominic Kao \\ MIT \\ 77 Massachusetts Ave \\ Cambridge, MA 02139 USA \\ dkao@mit.edu \\ D. Fox Harrell \\ MIT \\ 77 Massachusetts Ave \\ Cambridge, MA 02139 USA \\ fox.harrell@mit.edu
}

\begin{abstract}
The color red has been shown to hinder performance, motivation, and affect in a variety of contexts involving cognitively demanding tasks [16, 47, 49, 22, 11, 32, 44, 64, 41]. Teams wearing red have been shown to impair the performance of opposing teams [7, 28, 57, 21], present even in online gaming [27]. Although color is strongly contextual (e.g., red-failure association), its effects are posited to be sub-conscious [15] and operate powerfully even on nonhuman primates, e.g., Rhesus macaques (Macaca mulatta) take food significantly less often from an experimenter wearing red [39]. Here, we present one of the first studies on avatar color in a single-player game. We compared players using a red avatar to players using a blue avatar. Using the Game Experience Questionnaire (GEQ) [26], we find that players using a red avatar had a decrease in competence, immersion and flow. Our results are of consequence to how we design and choose colors in single-player contexts.
\end{abstract}

\section{Author Keywords}

Avatar Color; Educational Games; Virtual Identity; Avatars

\section{ACM Classification Keywords}

K.8.0 [Personal Computing]: General - Games 


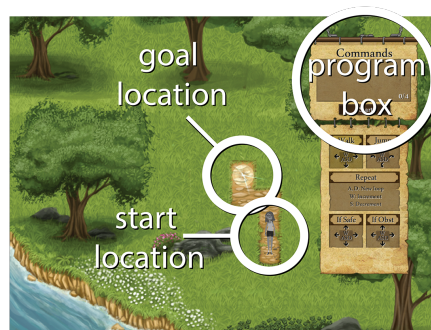

Figure 1: Level 1 in Mazzy introduces the basic game mechanics.

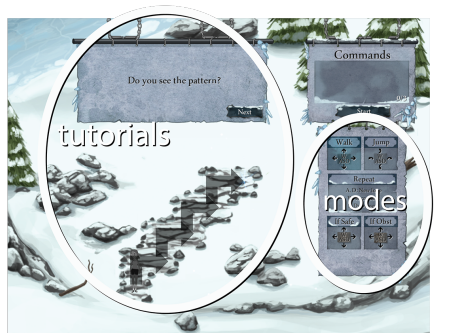

Figure 2: Level 6 introduces looping

\section{Introduction}

Over 120 years of research on color and its effects on humans have led to Color-In-Context (CIC) theory [10]. CIC has six premises: (1) Color carries meaning, i.e., color is more than aesthetics, (2) Color influences psychological functioning, e.g., colors are evaluated to be hospitable or hostile [3, 9, 66, 42], (3) Color effects are outside of conscious awareness [56, 40,45], (4) Color meaning is both learned and intrinsic, i.e., paired color associations such as pink is feminine; color vision as an adaptation [52, 29, 24, 4], (5) Color perception influences affect, cognition, and behavior, and vice versa [50, 19, 2], (6) Color is context-specific, e.g., pink is frequently viewed as feminine on a baby's blanket, but not on Bazooka bubble gum [65].

This gives us a framework for understanding how color may affect us in digital spaces. For example, most students in primary school are primed to associate red and failure in an evaluative context $[62,61,51,47]^{1}$. Moreover, red has associations with blood, danger, fire and anger. Red has been posited to be a distractor signal. Since Hill's seminal paper on the Athens Olympic Games in 2004 in which it was found that red-wearing competitors won more bouts than blue-wearing competitors in four different sports [21], there has been a plethora of research on color, motivation, and achievement. Later work found this work to be consistent in a variety of sporting domains [7, 28, 57, 21], and even in an online FPS game [27]

However, one gap in the literature is color in single-player contexts $^{2}$. To fill this need, we performed a study comparing players using a red avatar to players using a blue avatar,

${ }^{1}$ This association is not necessarily true across culture. For instance, an upward rise in China's stock market is represented in red $[67,30]$.

${ }^{2}$ Multiplayer studies on color exist $[27,5,58]$, as do a few on colored environments [54,31], but there are few studies on avatar color in singleplayer games. inside an educational game of our own creation. Although there is some question to whether, in the context of a sporting event, the color red is affecting the wearer, the opponents, or the referees, past work has consistently shown that red reduces mood, affect and performance in cognitiveoriented tasks [16, 47, 49, 22, 11, 32, 44, 64, 41]. For example, Lichtenfeld et. al showed that even just peripherally noticing red (e.g., hidden in a question, in the copyright notes at the end of a page, etc.) can have similar effects [44]. For this reason, we hypothesized that, if there were to be any effect on performance and game experience, that it would favor the blue avatar over the red avatar

\section{Motivation}

The work here is based on the premise that, along with factors such as subject mastery and affect toward the subject, a sense of identity as a STEM learner and doer is necessary for developing literacy and agency in computing [20]. The standard paradigm of computer-science education research traditionally focuses almost exclusively on cognitive challenges apparently inherent to particular computational concepts (e.g., [1]). Veeragoudar Harrell states that developments in the learning sciences suggest that computerscience curricula should embrace a broader conceptualization of learning: human reasoning, it is proposed, is embodied, distributed, and situated, and learning must be accordingly perceived as inherently collaborative, contextualized, and instrumented $[6,17,25,43,20]$. A result of this broader view of human reasoning and learning in the STEM disciplines is the emergence of research on relations between student identity and learning [18, 43, 53]. Recent work has shown that carefully selected colors can help induce student emotions that facilitate comprehension and transfer [60]. Digital manifestations of such phenomena are important areas for investigation since avatars are now fre- 


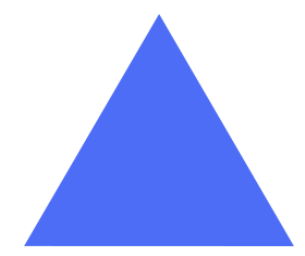

Figure 3: Blue Avatar.

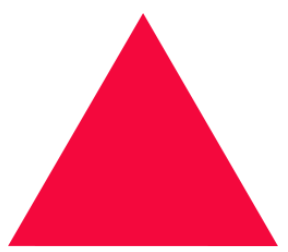

Figure 4: Red Avatar. quently used in videogames, Massive Open Online Courses (MOOCs) and forums, intelligent tutors, and more.

\section{The Game}

The experiment takes place in a STEM learning game called Mazzy [35]. Mazzy is a game in which players solve mazes by creating short computer programs. In total, there are 12 levels in this version of Mazzy. Levels 1-5 require only basic commands. Levels 6-9 require using loops. Levels 10-12 require using all preceding commands in addition to conditionals. See Figures 1 and 2. Mazzy has been used previously as an experimental testbed for evaluating the impacts of avatar type on performance and engagement in an educational game [38, 36, 34, 37, 33].

\section{Methods}

Our experiment aims to compare two colors of avatar: (a) blue avatar, and (b) red avatar. The goal is to see if participants using the two colors of avatar have differing game performance and game experience as measured by the GEQ. We strongly suspected ahead of time that results would favor the blue avatar.

\section{Avatar Conditions}

The two avatar conditions we tested were:

a. Blue Avatar

b. Red Avatar

The avatar was a triangle shape in both conditions, colored either blue or red. Color is defined by lightness, chroma, and hue. We keep lightness and chroma constant using the Munsell color system [12]. Only colors that can be displayed with good accuracy on a computer screen were considered ${ }^{3}$. The specific colors chosen were 7.5PB 5/18 (A)

${ }^{3} \mathrm{http}: / /$ www.andrewwerth.com/aboutmunsell/ and $5 R 5 / 18(\mathbf{A})$. See Figures 3 and 4 . Inside the game, the avatar consists of a $60 \times 60$ pixel game character that moves according to the user's programs.

Quantitative and Qualitative Measures

For performance, we only analyze the number of levels completed by players. For measuring game experience, we use the GEQ [26].

\section{Participants}

507 participants were recruited through Mechanical Turk. The data set consisted of 278 male, and 229 female participants. Participants self-identified their races/ethnicities as white (407), black or African American (29), Asian Indian (24), Chinese (5), Korean (4), American Indian (3), Vietnamese (3), Japanese (2), Filipino (1) and other (29). Participants were between the ages of 18 and $65(M=30.3$, $S D=8.7)$, and were all from the United States. Participants were reimbursed $\$ 1.50$ to participate in this experiment.

\section{Design}

A between-subjects design was used: avatar color was the between-subject factor. Participants were randomly assigned to a condition.

Protocol

Prior to starting the game, players were informed that they could exit the game at any time via a gray button in the corner of the screen. When participants were done playing (either by exiting early, or by finishing all 12 levels), participants returned to the experiment instructions, which then prompted them with the GEQ and then a demographics survey.

Analysis

Data was extracted and imported into Statistical Package for Social Science (SPSS) version 22 for data analysis us- 


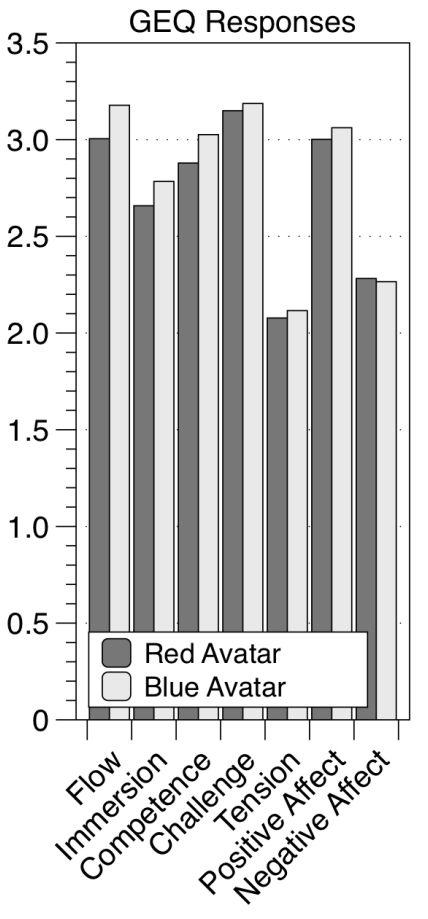

Figure 5: Game Experience Questionnaire (GEQ) responses for all participants. ing multivariate analysis of variance (MANOVA). The dependent variables were- GEQ items; and the independent variable was- avatar color (blue or red). All the dependent variables are continuous variables. The independent variable avatar color (i.e., 0 = blue, 1 = red) was a dichotomous variable. To detect the significant differences between blue avatar and red avatar, we utilized one-way MANOVA. We also ran an independent-samples t-test on the variable- levels completed. These results are reported as significant when $\mathrm{p}<0.05$ (two-tailed). Prior to running our MANOVA, we checked both assumption of homogeneity of variance and homogeneity of covariance by the test of Levene's Test of Equality of Error Variances and Box's Test of Equality of Covariance Matrices; and both assumptions were met by the data ( $p>.05$ for Levene's Test, and p>.001 for Box's Test).

\section{Results}

Aggregate

A MANOVA revealed a statistically significant difference in GEQ responses based on the participant's avatar color, $\mathrm{F}(42,464)=1.43, \mathrm{p}<.05$; Wilk's $\lambda=0.885$, partial $\eta^{2}=.12$. See Figure 5. Pair-wise comparisons revealed that the blue avatar GEQ rating was higher on "I was fully occupied with the game" (flow), $p=.015$, "It felt like a rich experience" (immersion), $\mathrm{p}=.018$, and "I felt competent" (competence), $\mathrm{p}=.044$. Blue was marginally higher on "I felt completely absorbed" (flow), $\mathrm{p}=.058$, "I forgot everything around me" (flow), $\mathrm{p}=.077$, "I lost track of time" (flow), $\mathrm{p}=.056$, "I felt imaginative" (immersion), $\mathrm{p}=.099$, "I felt that I could explore things" (immersion), $\mathrm{p}=.094$, "I felt skillful" (competence), $\mathrm{p}=.068$, "I was good at it" (competence), $\mathrm{p}=.059$, and "I felt successful" (competence), $p=.061$. The other dimensions (challenge, tension, affect) showed no significant differences. Levels completed by players using red (7.80) did not significantly differ from players using blue (7.74), $p$ $>0.05$.
Gender

We wanted to investigate if the previous differences appeared to affect both genders. From Figure 6, we can see that the general trend is the same as in Figure 5 for both genders (i.e., blue $>$ red across the three measures). However, the effect appears to be weaker in female participants. The effective difference in male participants compared to female participants is $12 x$ larger for flow, $3 x$ larger for immersion, and $1.3 x$ larger for competence. These results are consistent with literature that suggests red is more impactful on men [21, 23].

\section{Text Responses}

Using Linguistic Inquiry Word Count (LIWC) 2015 [59], we analyzed text responses of participants' answers to "Describe how you felt about your avatar". LIWC found that negative sentiment was significantly higher for players using the red avatar (6.09) than for players using the blue avatar (3.29), $t(503)=1.973, p<.05$. Positive sentiment in players using the red avatar (8.18) did not significantly differ from players using the blue avatar (8.85), $p>05$.

Were the colors hard to see?

To determine if the color negatively interacted with the game background, participants were asked "The avatar was hard to see" (1: Not at all, to 5: Extremely). Both blue participants $(\mathrm{M}=1.36, \mathrm{SD}=0.76)$ and red participants $(\mathrm{M}$ $=1.30, \mathrm{SD}=0.70$ ) had low scores, suggesting both avatar colors were clearly visible. Scores did not differ between the two conditions, $p>05$.

\section{Limitations}

Color stimuli varies on hue, lightness, and chroma. According to Elliot et. al [10], nearly all existing studies fail to control for these in color manipulations. This makes both interpretation and replication impossible. For example, the majority of research uses hues which the investigators believe 


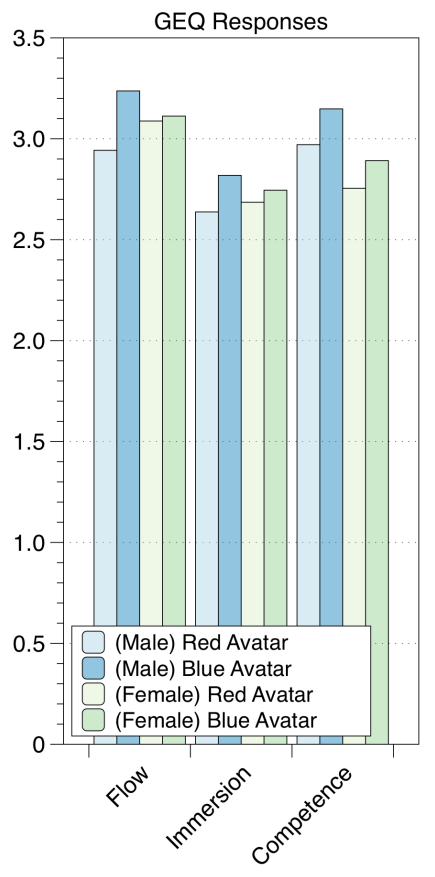

Figure 6: Game Experience Questionnaire (GEQ) responses for male and female participants. are the most ideal representatives. However, the problem is that this almost undoubtedly confounds color properties; for instance, "prototypical red" is more intense than "prototypical yellow".

The colors in this paper were selected from the Munsell color system, such that the following criteria were met: (1) the colors are equal in lightness and chroma, (2) the colors do not clash with the game interface, and (3) the colors are accurate on calibrated monitors.

Nonetheless, users each have their own individual monitors, graphic cards, and calibration settings. Not all users will see "exactly" the same color (as in a laboratory setting), but this approach strengthens external validity and reflects realistic applied settings. We do note that our participants were all from the U.S.

\section{Discussion}

We summarize our results:

- Blue led to higher flow than Red

- Blue led to higher immersion than Red

- Blue led to higher competence than Red

- Blue led to higher (avatar) affect than Red

Our results suggest that avatar color has significant effects on player flow, immersion, and competence. Although we have only investigated two of the colors most prevalent in literature [49], it's reasonable to hypothesize that other colors may also impact players. For instance, it was found in [31] that different colored environments may impact affect. To the best of our knowledge, this is one of the first studies to research the effects of avatar color in a single-player context. These results extend and support work on first-person shooter (FPS) multiplayer games in which it is hypothesized that blue teams are at a disadvantage because they "see red" [27].

In this study, we found that red had a negative effect on participant flow, immersion, competence, and avatar affect. Biologically, it has been hypothesized that the color red is a distractor signal to humans. Red causes a lower so-called high frequency heart rate variability (HF-HRV), measured via an electrocardiogram (ECG) [8]. These lower levels of HF-HRV are correlated to an increase in worry and anxiety $[14,13,49]$.

However, color is context-specific. Although the color red has been found to hinder motivation, performance, and affect in cognitive tasks [16, 47, 49, 22, 11, 32, 44, 64, 41], red has been shown to promote approach-like tendencies when in the context of "dating" [48]. The current investigation used as a setting a computer science learning game, and so it is reasonable to predict that red is hindering. Such effects may translate to changes in academic self-concept [63]. However, were the color red presented in the context of, e.g., a social game ([46, 55], etc.), it's possible that it's effects would be less negative.

\section{Conclusion}

This paper has explored the effects of avatar color. Our results suggest that the color of game avatars does impact player experience in significant ways. Game developers should be aware of the cognitive effects of using colors, both general as well as context-sensitive impacts. Information about general results, such as articulated in this paper in a case study considering our use of red, can lead educational game developers to make more informed choices about color in specific settings in light of user and developer needs and values. 


\section{Acknowledgments}

We would like to thank the anonymous reviewers for their valuable feedback. This work is supported by NSF STEM+C Grant 1542970 and a Natural Sciences and Engineering Research Council of Canada (NSERC) fellowship.

\section{References}

[1] Yehezkel Ben-Ari. 2001. Developing networks play a similar melody. (2001). DOI : http://dx.doi.org/10.1016/ S0166-2236(00)01813-0

[2] Emanuel Bubl, Elena Kern, Dieter Ebert, Michae Bach, and Ludger Tebartz Van Elst. 2010. Seeing gray when feeling blue? Depression can be measured in the eye of the diseased. Biological Psychiatry 68, 2 (2010), 205-208. DOI : http://dx.doi.org/10.1016/j. biopsych.2010.02.009

[3] John T. Cacioppo, W. L. Gardner, and G. G. Berntson. 1999. The affect system has parallel and integrative processing components: Form follows function. Journal of Personality and Social Psychology 76, 5 (1999), 839-855. http://citeseerx.ist.psu.edu/viewdoc/summary? doi $=10.1 .1 .94 .3238$

[4] Mark a Changizi, Qiong Zhang, and Shinsuke Shimojo. 2006. Bare skin, blood and the evolution of primate colour vision. Biology letters 2, 2 (2006), 217-221. DOI : http://dx.doi.org/10.1098/rsbl.2006.0440

[5] Ignacio X. Dominguez and David L. Roberts. 2015 Asymmetric Virtual Environments : Exploring the Effects of Avatar Colors on Performance. Experimental Artificial Intelligence in Games: Papers from the AIIDE Workshop (2015).

[6] Paul Dourish. 2001. Seeking a Foundation for ContextAware Computing. Human-Computer Interaction 16 (2001), 229-241. DOI : http://dx.doi.org/10.1207/ S15327051HCl16234\{_\}07
[7] Dennis Dreiskaemper, Bernd Strauss, Norbert Hagemann, and Dirk Büsch. 2013. Influence of red jersey color on physical parameters in combat sports. Journal of sport \& exercise psychology 35, FEBRUARY (2013), 44-9. http://www.ncbi.nlm.nih.gov/pubmed/ 23404878

[8] Andrew J Elliot and Henk Aarts. 2011. Perception of the color red enhances the force and velocity of motor output. Emotion (Washington, D.C.) 11, 2 (2011), 445449. DOI : http://dx.doi.org/10.1037/a0022599

[9] A J Elliot and Martin V Covington. 2001. Approach and avoidance motivation. Educational Psychology Review 13, 2 (2001), 73-92. DOI : http://dx.doi.org/10.1023/A: 1009009018235

[10] Andrew J. Elliot and Markus a. Maier. 2012. Color-inContext Theory. In Advances in Experimental Social Psychology. Vol. 45. 61-125. DOI : http://dx.doi.org/10. 1016/B978-0-12-394286-9.00002-0

[11] Andrew J Elliot, Markus a Maier, Arlen C Moller, Ron Friedman, and Jörg Meinhardt. 2007. Color and psychological functioning: the effect of red on performance attainment. Journal of experimental psychology. General 136, 1 (2007), 154-168. DOI : http://dx.doi.org/10.1037/0096-3445.136.1.154

[12] M D Fairchild. 2005. The Munsell book of color. In Color Appearance Models.

[13] Bruce H. Friedman. 2007. An autonomic flexibilityneurovisceral integration model of anxiety and cardiac vagal tone. Biological Psychology 74, 2 (2007), 185199. DOI : http://dx.doi.org/10.1016/j.biopsycho.2005.08. 009

[14] Bruce H. Friedman and Julian F. Thayer. 1998. Anxiety and autonomic flexibility: A cardiovascular approach. Biological Psychology 47, 3 (1998), 243-263. DOI : http://dx.doi.org/10.1016/S0301-0511(97)00027-6 
[15] Ronald S Friedman and Jens Förster. 2010. Implicit affective cues and attentional tuning: an integrative review. Psychological bulletin 136, 5 (2010), 875-893. DOI : http://dx.doi.org/10.1037/a0020495

[16] Timo Gnambs, Markus Appel, and Bernad Batinic. 2010. Color red in web-based knowledge testing. Computers in Human Behavior 26, 6 (2010), 16251631. DOI : http://dx.doi.org/10.1016/j.chb.2010.06.010

[17] James G Greeno, Allan M Collins, and Lauren B Resnick. 1996. Cognition and learning. Handbook of educational psychology. In Handbook of educational psychology. 15-46. http://psycnet.apa.org/psycinfo/ 1996-98614-001

[18] Melissa Gresalfi, Taylor Martin, Victoria Hand, and James Greeno. 2009. Constructing competence: An analysis of student participation in the activity systems of mathematics classrooms. Educational Studies in Mathematics 70, 1 (2009), 49-70. DOI : http://dx.doi.org/ 10.1007/s10649-008-9141-5

[19] Thorsten Hansen, Maria Olkkonen, Sebastian Walter, and Karl R Gegenfurtner. 2006. Memory modulates color appearance. Nature neuroscience 9, 11 (2006), 1367-8. DOI : http://dx.doi.org/10.1038/nn1794

[20] S Veeragoudar Harrell and Df Harrell. 2009. Exploring the Potential of Computational Self-Representations for Enabling Learning: Examining At-risk Youths' Development of Mathematical/Computational Agency. Proceedings of the Digital Arts and Culture Conference (2009).

[21] Russell a Hill and Robert a Barton. 2005. Psychology: red enhances human performance in contests. Nature 435, May (2005), 293. DOI : http://dx.doi.org/10.1038/ 435293a

[22] Bart Hulshof. 2013. The influence of colour and scent on people's mood and cognitive performance in meeting rooms. Master Thesis May (2013), 1-97.

[23] Anya C. Hurlbert and Yazhu Ling. 2007. Biological components of sex differences in color preference. Current Biology 17, 16 (2007), 623-625. DOI : http: //dx.doi.org/10.1016/j.cub.2007.06.022

[24] John Hutchings. 1997. Folklore and Symbolism of Green. Folklore 108 (1997), 55-63. DOI : http://dx.doi. org/10.1080/0015587X.1997.9715937

[25] Edwin Hutchins. 2000. Distributed Cognition. (2000). DOI : http://dx.doi.org/10.1007/s10111-004-0172-0

[26] W IJsselsteijn, Y De Kort, K Poels, A Jurgelionis, and Francesco Bellotti. 2007. Characterising and Measuring User Experiences in Digital Games. International Conference on Advances in Computer Entertainment Technology 620 (2007), 1-4. DOI : http://dx.doi.org/10.1007/978-1-60761-580-4

[27] Andrei llie, Silvia Ioan, Leon Zagrean, and Mihai Moldovan. 2008. Better to be red than blue in virtual competition. Cyber Psychology \& Behavior 11, 3 (2008), 375-377. DOI : http://dx.doi.org/10.1089/cpb. 2007.0122

[28] Silvia loan, Miriam Sandulache, Sinziana Avramescu, Andrei llie, Alexandru Neacsu, Leon Zagrean, and Mihai Moldovan. 2007. Red is a distractor for men in competition. Evolution and Human Behavior 28, 4 (2007), 285-293. DOI : http://dx.doi.org/10.1016/j. evolhumbehav.2007.03.001

[29] Gerald H. Jacobs. 1981. Comparative Color Vision. 159-182 pages. DOI : http://dx.doi.org/10.1016/ B978-0-12-378520-6.50010-8

[30] Feng Jiang, Su Lu, Xiang Yao, Xiaodong Yue, and Wing tung Au. 2014. Up or down? How culture and color affect judgments. Journal of Behavioral Decision Making 27, 3 (2014), 226-234. DOI : http://dx.doi.org/10.1002/bdm.1800 
[31] Evi Joosten, Gv Lankveld, and Pieter Spronck. 2010. Colors and emotions in video games. 11th International Conference on Entertainment Computing Figure 1 (2010).

[32] llyung Jung, MyungShik Kim, and Kwanghee Han. 2011. Red for Romance, Blue for Memory. In $\mathrm{HCl}$ International 2011 - Posters Extended Abstracts. Vol. 173. 284-288. DOI : http://dx.doi.org/10.1007/ 978-3-642-22098-2\{ \}57

[33] Dominic Kao and D. Fox Harrell. 2015a. Exploring construction, play, use of virtual identities in STEM learning. Jean Piaget Society Annual Conference (2015).

[34] Dominic Kao and D. Fox Harrell. 2015b. Exploring the Impact of Role Model Avatars on Game Experience in Educational Games. The ACM SIGCHI Annual Symposium on Computer-Human Interaction in Play (CHI PLAY) (2015).

[35] Dominic Kao and D. Fox Harrell. 2015c. Mazzy: A STEM Learning Game. Foundations of Digital Games (2015).

[36] Dominic Kao and D. Fox Harrell. 2015d. Toward Avatar Models to Enhance Performance and Engagement in Educational Games. IEEE Computational Intelligence in Games (2015).

[37] Dominic Kao and D. Fox Harrell. 2015e. Toward Evaluating the Impacts of Virtual Identities on STEM Learning. Foundations of Digital Games (2015).

[38] Dominic Kao and D. Fox Harrell. 2016. Toward Understanding the Impacts of Role Model Avatars on Engagement in Computer Science Learning. The annual meeting of the American Educational Research Association (AERA) (2016).

[39] Sara a Khan, William J Levine, Seth D Dobson, and Jerald D Kralik. 2011. Red signals dominance in male rhesus macaques. Psychological science : a journal of the American Psychological Society / APS 22, 8 (2011), 1001-1003. DOI : http://dx.doi.org/10.1177/ 0956797611415543

[40] Árni Kristjánsson, Patrik Vuilleumier, Paresh Malhotra, Masud Husain, and Jon Driver. 2005. Priming of color and position during visual search in unilateral spatial neglect. Journal of cognitive neuroscience 17 (2005), 859-873. DOI : http://dx.doi.org/10.1162/ 0898929054021148

[41] Christof Kuhbandner and Reinhard Pekrun. 2013. Joint effects of emotion and color on memory. Emotion (Washington, D.C.) 13, 3 (2013), 375-9. DOI : http://dx.doi.org/10.1037/a0031821

[42] Peter J Lang. 1995. The Emotion Probe. American Psychologist Association 50, 5 (1995), 372-385. DOI : http://dx.doi.org/10.1037/0003-066X.50.5.372

[43] Jean Lave and Etienne Wenger. 1991. Situated learning: Legitimate peripheral participation. Vol. 95.138 pages. DOI : http://dx.doi.org/10.2307/2804509

[44] Stephanie Lichtenfeld, Markus a. Maier, Andrew J. Elliot, and Reinhard Pekrun. 2009. The semantic red effect: Processing the word red undermines intellectual performance. Journal of Experimental Social Psychology 45, 6 (2009), 1273-1276. DOI : http://dx.doi.org/10.1016/.jesp.2009.06.003

[45] Jonathan Ling and Mark Blades. 2002. Further Evidence for Automatic Encoding of Colour by Children and Adults. British Journal of Developmental Psychology 20, 4 (2002), 537-544. DOI : http: //dx.doi.org/10.1348/026151002760390936

[46] Josh Mccoy, Mike Treanor, Ben Samuel, Brandon Tearse, Michael Mateas, and Noah Wardrip-fruin. 2010. Comme il Faut. Journal of Affective Disorders 40, 1-2 (2010), 3. DOI : http://dx.doi.org/10.1016/ 0165-0327(96)82444-X 
[47] Ravi Mehta and Rui(Juliet) Zhu. 2008. Blue or Red? Exploring the Effect of Color on Cognitive Task Performances. Science 323, February (2008), 1226-1229. DOI : http://dx.doi.org/10.1126/science.1169144

[48] Brian P. Meier, Paul R. D’Agostino, Andrew J. Elliot, Markus a. Maier, and Benjamin M. Wilkowski. 2012. Color in context: Psychological context moderates the influence of red on approach- and avoidancemotivated behavior. PLOS ONE 7, 7 (2012), 1-5. DOI : http://dx.doi.org/10.1371/journal.pone.0040333

[49] M.A. Meier, Russell A. Hill, Andrew J. Elliot, and R.A. Barton. 2015. Color in Achievement Contexts in Humans. Handbook of Color Psychology 44, February (2015), 0-103. DOI : http://dx.doi.org/10.1063/1.2756072

[50] Holger Mitterer, Jörn M Horschig, Jochen Müsseler, and Asifa Majid. 2009. The influence of memory on perception: it's not what things look like, it's what you call them. Journal of experimental psychology. Learning, memory, and cognition 35, 6 (2009), 1557-1562. DOI : http://dx.doi.org/10.1037/a0017019

[51] Arlen C Moller, Andrew J Elliot, and Markus a Maier 2009. Basic hue-meaning associations. Emotion (Washington, D.C.) 9, 6 (2009), 898-902. DOI : http: //dx.doi.org/10.1037/a0017811

[52] J D Mollon. 1989. "Tho' she kneel'd in that place where they grew..." The uses and origins of primate colour vision. The Journal of experimental biology 146 (1989), 21-38.

[53] Na'ilah Suad Nasir. 2002. Identity, Goals, and Learning: Mathematics in Cultural Practice. (2002). DOI : http://dx.doi.org/10.1207/S15327833MTL04023\{\}6

[54] Simon Niedenthal. 2007. Dynamic Lighting for Tension in Games. Game Studies (2007).

[55] Jeff Orkin and Deb Roy. 2007. The restaurant game: Learning social behavior and language from thou- sands of players online. Journal of Game Development 3, December (2007), 39-60. http://media.mit.edu/ cogmac/publications/Orkin

[56] Harshada Patel, Jackie Andrade, and Mark Blades. 2001. Children's incidental learning of the colors of objects and clothing. (2001). DOI : http://dx.doi.org/10. 1016/S0885-2014(02)00072-2

[57] Vincent Payen, Andrew J. Elliot, Stephen a. Coombes, Aïna Chalabaev, Jeanick Brisswalter, and François Cury. 2011. Viewing red prior to a strength test inhibits motor output. Neuroscience Letters 495 (2011), 4448. DOI : http://dx.doi.org/10.1016/j.neulet.2011.03.032

[58] J. Pena, J. T. Hancock, and N. a. Merola. 2009. The Priming Effects of Avatars in Virtual Settings. Communication Research 36, 6 (2009), 838-856. DOI : http://dx.doi.org/10.1177/0093650209346802

[59] J W Pennebaker, Ryan L. Boyd, Kayla Jordan, and Kate Blackburn. 2015. The Development and Psychometric Properties of LIWC2015. (2015).

[60] Jan L. Plass, Steffi Heidig, Elizabeth O. Hayward, Bruce D. Homer, and Enjoon Um. 2014. Emotional design in multimedia learning: Effects of shape and color on affect and learning. Learning and Instruction 29 (2014), 128-140. DOI : http://dx.doi.org/10.1016/j. learninstruc.2013.02.006

[61] Karyn Pravossoudovitch, Francois Cury, Steve G Young, and Andrew J Elliot. 2014. Is red the colour of danger? Testing an implicit red-danger association. Ergonomics 57, 4 (2014), 503-10. DOI: http://dx.doi.org/10.1080/00140139.2014.889220

[62] Abraham M Rutchick, Michael L Slepian, and Bennett $D$ Ferris. 2010. The pen is mightier than the word: Object priming of evaluative standards. European Journal of Social Psychology 40 (2010), 704-708. DOI : http://dx.doi.org/10.1002/ejsp.753 
[63] Michael James Scott and Gheorghita Ghinea. 2013. Integrating Fantasy Role-Play Into the Programming Lab. Proceeding of the 44th ACM Technical Symposium on Computer Science Education - SIGCSE '13 (2013), 119. DOI : http://dx.doi.org/10.1145/2445196. 2445237

[64] Jiaxin Shi, Chao Zhang, and Feng Jiang. 2015. Does red undermine individuals' intellectual performance? A test in China. International Journal of Psychology 50, 1 (2015), 81-84. DOI : http://dx.doi.org/10.1002/ijop. 12076
[65] S. E. Ural and S. Yilmazer. 2010. The architectural color design process: An evaluation of sequential media via semantic ratings. Color Research and Application (2010).

[66] R. B. Zajonc. 1998. Emotions. In The handbook of social psychology. 591-632.

[67] Tengxiao Zhang and Buxin Han. 2014. Experience reverses the red effect among Chinese stockbrokers. PloS one 9, 2 (jan 2014), e89193. DOI : http://dx.doi.org/ 10.1371/journal.pone.0089193 